Argumentation et Analyse du Discours

\title{
De la contestation doxique dans le champ discursif de l'école française
}

Some examples of doxic dissent in the discursive field of the French school

Pascale Delormas

\section{OpenEdition}

1 Journals

Édition électronique

URL : http://journals.openedition.org/aad/2404

DOI : 10.4000/aad.2404

ISSN : $1565-8961$

Éditeur

Université de Tel-Aviv

Référence électronique

Pascale Delormas, "De la contestation doxique dans le champ discursif de l'école française », Argumentation et Analyse du Discours [En ligne], 19 | 2017, mis en ligne le 15 octobre 2017, consulté le 23 septembre 2019. URL : http://journals.openedition.org/aad/2404; DOI : 10.4000/aad.2404

Ce document a été généré automatiquement le 23 septembre 2019.

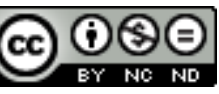

Argumentation \& analyse du discours est mis à disposition selon les termes de la licence Creative Commons Attribution - Pas d'Utilisation Commerciale - Pas de Modification 4.0 International. 


\title{
De la contestation doxique dans le champ discursif de l'école française
}

Some examples of doxic dissent in the discursive field of the French school

\author{
Pascale Delormas
}

1 L'observation des lieux de tension propres au champ discursif de l'école est susceptible de faire mieux comprendre la complexité de l'institution scolaire en France. En effet, celle-ci est structurée et vit, comme toute communauté en contexte démocratique (Amossy 2014), des dissensions et des ralliements idéologiques. La spécificité des différents positionnements en relation de concurrence dans le domaine de l'école sera examinée ici à travers quelques cas de figure exemplaires des controverses suscitées par l'expression de la doxa. Parole d'autorité susceptible de façonner les opinions et d'orienter les politiques, celle-ci provoque des réactions que l'on s'efforcera de décrire en référence au cadre théorique de l'analyse du discours ${ }^{1}$.

2 Nous entendons décrire les modalités d'expression du dissensus à travers trois genres de discours sélectionnés en fonction des différentes phases de « diffusion doxique » ou «modes de variation expressive du sens commun ${ }^{2}$ » que distingue Sarfati (2007) : celles du canon, de la vulgate et de la doxa. En l'occurrence, nous nous proposons d'observer les caractéristiques d'un rapport de l'Inspection générale ${ }^{3}$ qui remet en cause de manière explicite et argumentée la conception des Instructions officielles de l'enseignement secondaire de l'Histoire et géographie (phase du canon), celles d'écrits réflexifs de futurs enseignants que nous envisageons comme contestation implicite et oblique d'une contrainte curriculaire (phase de la vulgate) et, enfin, une intervention polémique de Nicolas Sarkozy, Président de la République, filmée, largement diffusée et perçue comme un désaveu infligé à la communauté des gens cultivés (phase de la doxa).

Ces discours doivent être mis en relation avec la situation dans laquelle se trouvent les locuteurs ${ }^{4}$. Il est nécessaire pour cela de se référer à l'interdiscours dont ils participent, c'est-à-dire de convoquer les discours auxquels ils s'opposent ou qu'ils suscitent, en lien avec les choix génériques et énonciatifs contraints par le statut des coénonciateurs. 


\section{Contestation du canon dans un rapport de l'Inspection générale : une critique argumentée des Instructions officielles}

4 Le genre du rapport de l'Inspection générale n'est pas soumis aux mêmes règles que les textes de cadrage, c'est-à-dire les Instructions officielles (ou "programmes»), auxquelles ils se réfèrent et qui fixent le canon. Il doit être abordé à travers la relation étroite qu'il entretient avec les programmes souvent remaniés, depuis les objectifs jusqu'aux contenus, et dont les préconisations sont censées accompagner une évolution sociétale, voire politique.

\subsection{Instructions officielles et rapport d'inspection : des genres discursifs différents}

Les Instructions officielles ne supposent pas la mise en œuvre d'une argumentation : les éventuels débats sont censés avoir eu lieu en amont. Leur vocation est d'indiquer des orientations pédagogiques et didactiques sur la durée en établissant objectifs et exigences; ils sont l'expression d'une décision qui doit s'imposer aux enseignants soumis à la hiérarchie, si bien que c'est la modalité prescriptive qui est attendue ${ }^{5}$. En revanche, les rapports de l'Inspection générale ${ }^{6}$ interviennent en aval. Ils sont définis comme le résultat d'une expertise qui doit être remise chaque année au Ministre en vue d'assurer «le suivi permanent de la mise en œuvre de la politique éducative » dans le but de «contribuer à la prise de décisions des responsables concernés ${ }^{7}$. La critique constructive et argumentée est donc de mise.

Un bon exemple d'absence de consensus parmi les personnels chargés de définir des orientations des enseignements nous est donné à travers un rapport de l'Inspection générale d'histoire et géographie de 2010 à propos de l'éducation à la citoyenneté européenne ${ }^{8}$. La place accordée à l'Europe dans les programmes d'histoire, de géographie et d'éducation civique aux différents niveaux de la scolarité (collège, lycée d'enseignement général et lycée professionnel) y est remise en cause dès le préambule. Ainsi peut-on lire en introduction que

[1]es programmes actuels intègrent une réelle dimension européenne mais très orientée sur l'Union européenne au détriment du reste des États européens, notamment ceux de l'Europe médiane et orientale. C'est très net en collège comme au lycée, même si les programmes reflètent les hésitations, tant scientifiques que politiques, face au mouvement d'intégration européenne.

(http://www.emilangues.education.fr/references/etudes-et-rapports)

7 L'état des lieux qui suit ce paragraphe n'est pas purement descriptif: la partie consacrée à «L'Europe dans les Programmes » engage un jugement dès les intertitres («L'Europe dans les programmes de collèges: une entrée privilégiée » / "L'Europe dans les programmes du lycée d'enseignement général : le tournant des programmes de 1995 ») même si les auteurs admettent, dans le développement, une orientation positive ( «il y a là une évolution nouvelle qu'il faut souligner » / "l'Europe est mieux prise en compte " / "L'Europe devient un véritable objet d'étude») parfois sur un mode concessif ( «le programme d'histoire concerne le monde du milieu du $18^{\text {ème }}$ siècle à 1939 ; l'Europe cependant est souvent placée au premier plan» / «[...] en géographie cependant, les programmes prennent acte de la nouvelle hiérarchie des puissances en 
inscrivant l'étude de l'Allemagne.»), modalisant ainsi un jugement parfois plus radical ( [...] les derniers allégements affaiblissent la dimension culturelle européenne du programme »). Puis la critique se fait plus virulente à travers des questions rhétoriques («Quelle vision de l'Europe est proposée aux élèves? / Y a-t-il des relations entre les représentations de l'Europe et le contexte politique ?) et une condamnation claire qui souligne les carences dans la conception des programmes («Un objet d'étude mal défini») voire l'incompétence des personnels institutionnels («Les hésitations des universitaires face à un concept qu'ils ne reconnaissent guère " / "L'Europe prétexte dans les projets d'établissement », ou encore : «En éducation civique, les professeurs et leurs élèves peinent à se percevoir comme citoyens - ou futurs citoyens - européens tant la citoyenneté leur apparaît indissociable de l'appartenance à un État-nation. »)

Quant à la « mise en œuvre des contenus européens des programmes dans les collèges et dans les lycées ", l'Inspection générale

déplore [...] l'approche traditionnelle, accumulatrice, sans interrogation ("un continent qui va de l'Atlantique à l'Oural "). On échappe rarement aux chapitres sur le relief et les climats, l'unité et la diversité. » / "Les États au programme sont souvent étudiés pour eux-mêmes et ne sont pas replacés dans l'organisation de l'espace européen, [...]. C'est notamment le cas pour la France, abordée le plus souvent dans sa stricte dimension hexagonale.» / "L'Europe est trop souvent réduite à l'Union européenne et aux étapes de sa construction. »

9 La formation des enseignants est également remise en cause car "l'Europe n'est jamais un axe important dans les plans de formation » et, en section européenne, elle serait «balbutiante ». Cependant, des « réalisations locales [sont] signalées», comme autant de «réponses [individuelles] aux demandes institutionnelles»en dépit des manquements au contrat ${ }^{9}$ :

Heureusement, quelques professeurs adoptent des démarches plus pertinentes [...] Un peu plus fréquentes sont les leçons où on utilise plus ou moins habilement les concepts de centre et de périphérie, de maillage et de treillage, tout cela au hasard des propositions des manuels et des cahiers de travaux pratiques consacrés à l'Europe.

\subsection{Un conflit de valeurs}

Sommés de répondre à des exigences complexes voire contradictoires, les concepteurs des programmes travaillent à effacer les marques d'énonciation les plus visibles, comme il convient dans un discours instituant. Cependant les spécialistes de la discipline et les fonctionnaires ministériels sont en mesure d'y déceler la diversité des points de vue et d'en comprendre les enjeux comme en témoignent le rapport de l'Inspection : il fait apparaître que les valeurs qui sous-tendent les Programmes, loin d'être stabilisées, sont en débat, en référence "à une mémoire qui, en retour, est ellemême prise dans les conflits du champ, qui ne cessent de la retravailler" (Maingueneau 2010: 88). Ce constat est ici d'actualité étant donné «les évolutions récentes qui reflètent la porosité croissante des politiques éducatives, de plus en plus influencées par d'autres politiques publiques, comme les politiques d'intégration " (Legris 2010, à propos des programmes d'histoire) et si l'on considère le fait que

l'ouverture du "circuit d'écriture" des programmes à des acteurs toujours plus nombreux complexifie la conception du citoyen véhiculée par ces textes [car] les programmes ne sont pas seulement un objet de concurrence entre historiens à 
propos de la construction de la nation, ils constituent également un enjeu politique et symbolique, plus ou moins brûlant selon les époques (idem).

11 En revanche, le rapport d'inspection que nous examinons manifeste une véritable auctorialité et si le dispositif énonciatif repose également sur l'effacement énonciatif attendu dans un discours institutionnel, il mentionne tout de même un point de vue auquel le caractère explicitement unanime doit donner du poids ( « les observations des inspecteurs se rejoignent pour ... »). Il signale un positionnement dans le champ dont le but affiché est de réunir une communauté autour de valeurs dont les garants sont défaillants. Les signataires s'opposent à une vision politique du monde qu'ils ne partagent pas et ils appellent de leurs vœux un tout autre type de relation à la question européenne que celle que prônent les Programmes.

Ainsi prédomine l'emploi d'un lexique critique de la politique libérale (« marchandisation », " marketing territorial»). Il est question de « la traditionnelle approche économiste dominante depuis Pierre George et son Europe des marchands, dans les années soixante ", du fait qu' " exceptionnelles sont les leçons qui évoquent la place de l'Europe sur l'échiquier mondial et sa capacité à contester la domination de la superpuissance américaine "; le rapport conclut de façon ironique qu' «au total, l'enseignement de la géographie de l'Europe reste encore trop traditionnel, trop nomenclatural, insuffisamment problématisé, sauf à considérer que les chapitres consacrés aux succès d'Airbus et d'Ariane constituent une approche positive ».

Le rapport se fait plus constructif lorsqu'il fait état de la possibilité d'un changement («

La construction d'une véritable conscience européenne est en gestation " / " la réalité même de l'Union européenne s'inscrit dans un mouvement historique encore inachevé ») et de la conviction selon laquelle il s'agit, plus fondamentalement, de promouvoir une "éducation au doute, au questionnement», une "inquiétude créatrice », car " l'identité culturelle européenne s'affirme dans le refus d'admettre une identification achevée, par conséquent dans l'incertitude et l'inquiétude ", autant d'affirmations étayées par l'évocation de travaux de recherche (de Michel Foucher, de Jean-Pierre Rioux, de Jacques Lévy et d'historiens médiévistes).

Ces actes de langage représentatifs (assertions configurant le monde tel qu'il est perçu) et directifs (programmatiques) reposent sur les mêmes caractéristiques de l'ethos discursif que celui que Rabatel (2015) relève dans le genre du manifeste. Il s'agit d'un ethos

légitime, sérieux, raisonnable (comme l'indiquent les concessions), motivé, rationnel, pondéré (comme le soulignent les explications très articulées des conséquences du système, l'absence de recherche de la formule, de la petite phrase), d'un ethos convaincu, appelant à l'adhésion en faveur d'un changement dont la finalité est le changement du cours des choses. En cela, il s'affirme comme un contre discours rationnel, fédérateur, légitime, qui conteste la légitimité de la logique dominante [...].

\section{Contestation de la vulgate dans les écrits réflexifs en formation des enseignants : une stratégie d'évitement}

15 L'écrit réflexif est un écrit prescrit dans le curriculum de la formation des maîtres en France. Il s'agit d'un hypergenre, c'est-à-dire d'une catégorie discursive peu contrainte 
(Maingueneau 2005), d'abord parce que l'autonomie des Écoles Supérieures du Professorat et de l'Éducation ${ }^{10}$ et la liberté pédagogique autorisent des orientations différentes : un cadrage national existe mais chaque centre de formation ${ }^{11}$ est habilité à configurer la prescription initiale en fonction de problématiques locales et chaque formateur est susceptible de faire le choix d'orienter les futurs enseignants qui lui sont confiés vers un écrit dont l'ethos discursif privilégiera celui du professionnel, celui du chercheur ou encore celui de la personne (Delormas 2012b, 2015) ${ }^{12}$.

Si l'institution réclame des "écrits réflexifs », c'est que ce sont des lieux privilégiés de légitimation. La réalisation de tels écrits durant la formation des enseignants tente de concilier deux objectifs : celui de la socialisation par la reproduction et celui de la constitution de l'individu en sujet professionnel. La légitimité des acteurs est en effet référée à la formation discursive dans laquelle ils se trouvent pris et non à une quelconque subjectivité et le discours institutionnel auquel ils sont censés recourir constitue la médiation sociale qui leur permet d'accéder à la conscience d'une identité collective ${ }^{13}$. Or les bilans des formateurs montrent que la face positive de l'étudiant (Brown et Levinson $1978: 61)^{14}$, en cause dans l'épreuve, « oriente l'effort vers la parade plutôt que vers la remise en cause des manières de faire » (Rayou 2002 : 30):

- les productions sont très (trop) centrées sur l'atteinte des objectifs programmatiques et ne questionnent pas suffisamment ce qui fait enseignement.

- les écrits présentés relatent une séquence ou un projet globalement réussi (perçu comme tel), il serait intéressant de voir en réflexion des situations plus problématiques (dans les deux sens du terme). ${ }^{15}$

17 Les écrits réflexifs construisent en effet une image de soi lisse, dénuée de l'inquiétude et du doute qui devraient caractériser l'individu en formation. Si la perfection professionnelle visée semble atteinte - si l'on en croit les nombreux énoncés déclaratifs - les traits de réflexivité sont ambigus. En voici trois extraits :

Extrait $1:$ L'atelier de pratique professionnelle sur la question du graphisme en fin de moyenne section, très formateur, a été complété par le livre clair et concis d'é. Villard [...]. J'ai pu mener un travail tout au long de l'année car je n'avais que sept moyennes sections l'après-midi et je pouvais suivre leur façon d'écrire et les guider. [...] Pour organiser mon travail, j'ai donc profité de la formation en IUFM et des outils qui m'avaient été donnés en début d'année.

L'éloge de la formation reçue, l'affirmation du principe selon lequel l'erreur est source de progrès, la manifestation de la conviction d'avoir rempli la mission d'accompagnement et de guidage qui incombe à l'enseignant comme celle de la pertinence des choix de lecture autonome sont des «éléments de langage » en totale conformité avec la demande institutionnelle.

Extrait 2: Les discussions, les expériences et le questionnement incessant ont permis de remédier à cette difficulté et a rendu cohérente ma façon de prendre en compte la diversité des élèves. [...] Si j'ai dû faire des efforts d'adaptation quant à mon langage, en maternelle [...] je pense avoir globalement acquis la compétence correspondant à maîtriser la langue française pour enseigner et communiquer [...]

Le mouvement réflexif semble confondu avec l'éloge de soi. Ainsi, l'ouverture, le goût de l'effort, la capacité d'adaptation sont mis en avant pour une autoévaluation souvent très positive. Les appréciations manquent parfois leur but tant l'absence de nuances contrevient au but recherché, comme c'est le cas dans l'extrait qui suit.

Extrait 3: Tout au long de l'année, j'ai gardé la volonté de m'améliorer (lecture d'ouvrages spécialisés, analyse de mes pratiques de classe) et mes difficultés m'ont été utiles pour mesurer mes progrès, pour aller plus loin grâce à la formation reçue. 
[...] J'ai pendant cette année modifié mes conceptions et la façon de mettre en œuvre mon enseignement. contestation ne peut se manifester ouvertement puisque l'obtention du diplôme dépend de l'évaluation de la formation. Sans doute sont-ils imputables à trois types de difficulté : la définition peu précise du genre "écrit réflexif ", l'ambiguïté du double statut d'enseignant et d'étudiant, la confrontation à la dimension idéologique dont ce type d'écrit est chargé. Chacun de ces aspects doit être développé.

21 1) Les étudiants déplorent le caractère flou du cadrage institutionnel : l'ethos visé dans l'écrit réflexif se trouve affecté du fait des interprétations diverses de la commande. D'autre part la notion même de réflexivité auquel cet écrit fait référence ne renvoie pas à une définition homogèn $\mathrm{e}^{16}$. Un extrait d'échanges sur un forum ${ }^{17}$ montre l'incertitude dans laquelle les étudiants sont plongés.

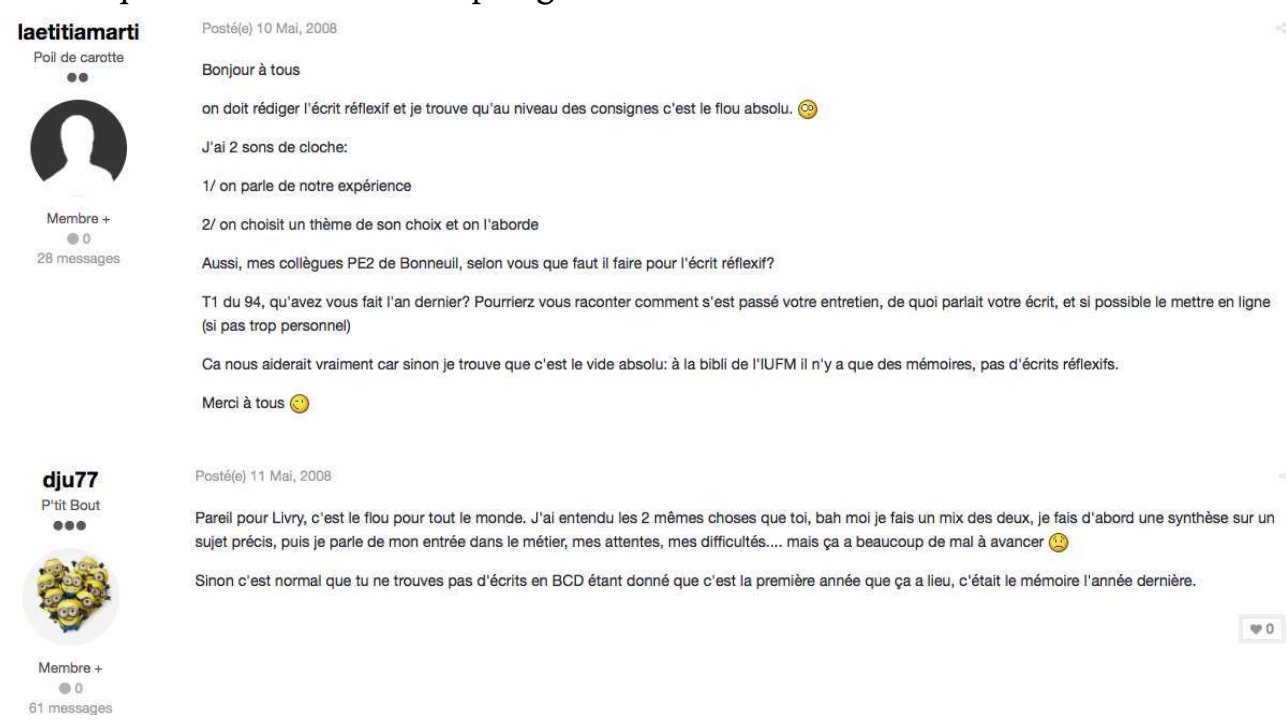

Les étudiants ne sont pas rassurés par leurs formateurs qui manifestent également leur désarroi. Ainsi peut lire sur une page d'un site très fréquenté les mots suivants :

Elle était attendue cette note du 28 octobre 2015 dans les ESPE, un cadrage «bienvenu» des attentes du "mémoire » qualifié «d'écrit scientifique réflexif » dans le texte. Attendue car en débat dans la plupart des écoles supérieures, les orientations comme les règles formelles ou encore les contenus ont, depuis la mastérisation, pris des formes variées attestant de l'importance variable et de l'usage formatif qui étaient dévolus à cet écrit.

La note du ministère éclaire une partie des arbitrages à produire mais laisse aussi en suspens beaucoup de questionnements. Entre volonté d'harmonisation et d'autonomie des structures et projets le chemin étroit n'est peut-être pas au service de la clarification des attendus. [...]

L'appellation "écrits scientifiques réflexif" ${ }^{18}$ est retenue pour qualifier les productions écrites réalisées par les différents publics de Master $\mathrm{MEEF}^{19}$ en fin de diplôme. Mais que traduisent ces qualificatifs de "scientifique et réflexif » présents dans la note ?20

2) La formation par alternance réclame que l'individu en formation endosse tantôt le rôle de l'enseignant sur le terrain face à une classe et tantôt celui de l'étudiant face à ses formateurs. Selon la situation, la hiérarchie des cadres primaire et secondaire (Goffman 1991) s'inverse: face à leurs formateurs, les futurs enseignants sont des apprenants et le cadre secondaire est la situation de classe. Face aux élèves, le stagiaire 
endosse l'ethos du maittre et le cadre de la formation constitue le cadre secondaire. Or l'écrit réflexif bouleverse cette hiérarchie et brouille la représentation de l'image de soi à construire.

3) Alors que les concepteurs du curriculum, négligeant l'ambiguïté qui le caractérise, voient dans l'écrit réflexif un exercice de construction de soi, nous pensons, dans la perspective de la subjectivation théorisée par Foucault (2001:1452) pour lequel est centrale « la manière dont le sujet fait l'expérience de lui-même dans un jeu de vérité où il a rapport à soi ", que, étant donné qu'elle touche le for privé, cette écriture sous contrainte risque d'inhiber toute velléité de travailler à la construction de l'ethos attendu par l'institution : elle amène souvent au refus de se soumettre à une demande jugée abusive et interprétée comme «interpellation » de l'individu en sujet (Althusser 1976 : 113-114) :

Nous suggérons alors que l'idéologie « agit » ou « fonctionne » de telle sorte qu'elle «recrute » des sujets parmi les individus (elle les recrute tous), ou «transforme » les individus en sujets (elle les transforme tous) par cette opération très précise que nous appelons l'interpellation, qu'on peut se représenter sur le type même de la plus banale opération policière (ou non) de tous les jours : « hé, vous, là-bas!». Si nous supposons que la scène théorique imaginée se passe dans la rue, l'individu interpellé se retourne. Par cette simple conversion physique de 180 degrés, il devient sujet. Pourquoi ? Parce qu'il a reconnu que l'interpellation s'adressait «bien » à lui, et que « c'était bien lui » qui était interpellé (et pas un autre). ${ }^{21}$

Cette demande peut être perçue également comme «technique de contrôle du comportement » (Foucault 2004) ${ }^{22}$. Ainsi, l'exercice de l'écriture réflexive aurait pour objectif d'améliorer les compétences et de développer les performances professionnelles des individus en les amenant à intérioriser des exigences externes et à assumer une responsabilisation morale comme technique de "gouvernementalité néolibérale $»^{23}$. Une telle prescription serait révélatrice de la diffusion de la conscience technocratique: elle annoncerait une rationalisation généralisée des pratiques exerçant une pression permanente sur tous les domaines de l'existence jusqu'aux plus intimes (armée, système scolaire, famille, etc.) (Wrana 2006 : 244).

\section{Contestation politico-médiatique de la doxa : l'enseignement littéraire au centre d'une polémique}

Les questions techniques restent en général confidentielles: elles relèvent de la compétence des spécialistes et ne mobilisent en principe que les professionnels de l'éducation. Or, on constate qu'elles sont parfois massivement relayées par des discours politico-médiatiques (Burger et al. 2011). Dès lors, le commentaire doxique n'est plus " contenu » par le cadre institutionnel comme c'est le cas pour les discours "fermés » (Maingueneau 1992: 120)24 : les discours politico-médiatiques en déplacent les enjeux; reflets aussi bien qu'agents de l'opinion publique, ils infléchissent indirectement les choix politiques qui façonnent l'image de l'école et de ce fait ils participent à la construction de sa réalité.

On considèrera ici un événement qui a fait couler beaucoup d'encre en France. Il s'agit de la remise en cause par un élu de la République de l'enseignement de la littérature dans le cadre des concours administratifs destinés aux professionnels dans le cadre de 
la "validation des acquis par l'expérience " ${ }^{25}$. C'est à l'occasion d'une intervention filmée en juillet $2008^{26}$ que le Président N. Sarkozy s'exprime dans les termes suivants :

Lorsque l'on parle des concours administratifs dans un parcours au mérite, on doit tenir compte, me semble-t-il, du fait que s'il y a deux candidats et il y a en un qui a fait 15 ans de service de bénévolat et l'autre qui a rien fait, on critique pas. On critique pas. Mais ça doit donner des points de plus à celui qui a fait du bénévolat pour les autres. Quand-même, je veux dire, en termes de richesse humaine, d'engagement aux services des autres. Pourquoi on n'en tiendrait pas compte? Ça vaut autant que de savoir par cœur La Princesse de Clèves. Enfin ... je n'ai rien contre, enfin, bon, enfin... c'est parce que j'ai beaucoup souffert sur elle ${ }^{27}$.

Les analyses de Grinshpun (2013a, 2013b) s'intéressent à la circulation de ces discours et aux manifestations langagières que la saillie présidentielle a suscitées dans la population et dans les différentes communautés en cause (diffusion massive sur les réseaux sociaux, manifestations dans la rue et dans la presse papier, émissions de slogans, etc.). Notre propos est d'interroger la dimension idéologique de cet événement médiatique à visée politicienne, étant donné le retentissement national que cette remise en cause de l'intérêt sociétal de l'enseignement de la littérature a connu.

Bafouant la légitimité de la doxa, N. Sarkozy manifeste son opposition à des valeurs promues par les « humanistes » alors qu'il devrait, à leurs yeux, en être le promoteur (Delormas 2012a). Deux traits majeurs apparaissent dans cette contestation transgressive selon laquelle la littérature ne servirait qu'à une classe de nantis : un exemplum particulièrement efficace et un ethos sincère. En effet, l'évocation de la fiction d'un roman galant devant des travailleurs engagés dans la vie professionnelle produit un effet cocasse et fait émerger la nécessité de la reconnaissance de l'expérience comme une évidence.

N. Sarkozy en "avouant » ses propres difficultés à s'approprier une culture lettrée manifeste de la compassion pour " la France qui se lève tôt $»^{28}$. Outre l'empathie dont elle fait preuve, l'efficacité de son intervention s'appuie sur une prosodie et une mimogestuelle dramatisantes que soutiennent les choix sémantiques. La réaction de ceux qui l'écoutent (rires de complicité) ne fait que renforcer le clivage entre les « héritiers » et les autres : le propos est en effet prétexte à séduire une certaine frange de la population qui se sentirait exclue des humanités à travers la provocation d'un autre groupe social, qui a, lui, la jouissance de ce capital culturel. Car l'adresse est double: au-delà de l'auditoire premier (les individus concernés par les concours) convaincu par une rhétorique que l'on peut qualifier de populiste, l'auditoire visé est celui des humanistes et des lettrés, supposé s'opposer au premier dans ses choix culturels. En lui opposant une évidence qu'elle ne partage pas, N. Sarkozy s'aliène une partie de la population mais se range à l'opinion la plus commune.

\section{Conclusion}

31 Analyser les discours de l'école suppose que l'on tienne compte des discours contestataires parce qu'ils participent pleinement à la reconfiguration permanente de cette institution. Les questions techniques dont s'emparent, à tous les niveaux, acteurs institutionnels, praticiens et médias pour en discuter le bien fondé à différents moments de diffusion doxique (canon, vulgate, doxa) sont en fait des questions d'ordre idéologique et politique. Cet article a tenté de faire apparaitre les configurations 
discursives qui en témoignent à travers l'analyse des stratégies adoptées par différents acteurs investis dans le champ de l'école.

Pour appréhender les discours contestataires qui se manifestent dans l'univers discursif de l'école, nous avons pris en considération les fonctions que le sémioticien Marin (1993) assigne aux représentations. La fonction de "représentation collective " de l'institution scolaire «organise les schèmes de classement, d'actions et de jugements", la fonction d'" exhibition» renvoie aux "rituels, stylisation de vie et signes symboliques qui les donnent à voir ", la fonction de "présentification ", à une "forme d'incarnation dans un représentant d'une identité collective» (cité par Charaudeau $2002: 504$ ).

Selon les rôles qu'assument les acteurs - ici, des inspecteurs généraux, des enseignants en formation, des politiciens - , selon les places qu'ils occupent et selon les opinions qu'ils soutiennent, les modalités de mise en scène du dissensus à travers lesquels ils activent leurs points de désaccord en radicalisant parfois leurs positionnements renvoient à la critique argumentée, à la rébellion implicite ou à la polémique - en l'occurrence, sur les scènes génériques du rapport d'inspection, de l'écrit réflexif et de l'intervention politico-médiatique. Enfin, le traitement de la question européenne dans l'enseignement, la construction de l'ethos enseignant dans la formation des maitres, la Princesse de Clèves comme métonymie de la littérature sont autant d'objets sur lesquels se cristallise la confrontation pour dénoncer certaines dérives comme la conception marchande de l'Europe, un gouvernement technocratique qui menace les libertés individuelles ou l'acculturation aux " humanités », envisagée comme marqueur social.

\section{BIBLIOGRAPHIE}

Althusser, Louis. 1976. «Idéologie et appareils idéologiques d'État (notes pour une recherche) », Positions (Paris : Editions Sociales)

Amossy, Ruth. 1999. Images de soi dans le discours (Paris : Nathan)

Amossy, Ruth. 2014. Apologie de la polémique (Paris : PUF)

Brown, Penelope \& Stephen C. Levinson. 1987. Politeness: Some universals in language usage

(Cambridge : Cambridge U. P.)

Attali, Alain. 1995. «L'inspection générale de l'éducation nationale », Revue internationale d'éducation de Sèvres 8, 57-65

Burger Marcel Jérôme Jacquin \& Raphaël Micheli. 2011. « L'analyse de la confrontation dans les discours politico-médiatiques contemporains ", Burger Marcel, Jérôme Jacquin \& Raphaël Micheli (éds), La parole politique en confrontation dans les médias (Bruxelles : De Boeck), 7-24

Charaudeau, Patrick. 2002. « Représentation sociale », Charaudeau, Patrick \& Dominique Maingueneau (éds), Dictionnaire d'analyse du discours (Paris : Seuil), 502-505

Crinon, Jacques \& Michèle Guigue. 2006. « Écriture et professionnalisation », Revue française de pédagogie 156, 117-169 
Delormas, Pascale. 2012a. « Interdiscours scolaire et légitimité de la lecture littéraire », $13^{\text {émes }}$ rencontres des chercheurs en didactique de la littérature, Université de Cergy-Pontoise, 29-31 mars : École et patrimoines littéraires : quelles tensions, quels usages aujourd'hui ? Communication non publiée.

Delormas, Pascale. 2012b. " Contribution à une analyse de l'ethos discursif de l'enseignant ", Pratiques 153/154, Les Littéracies universitaires : perspectives internationales, 100-114

Delormas, Pascale. 2015. «Das Genre reflexiven Schreibens in der Lehrer_innenbildung. Eine diskursanalytische Studie », Fegter, Susann, Kessl, Fabian, Langer, Antje, Ott, Marion, Rothe, Daniela, Wrana Daniel (éds), Erziehungswissenschaftliche Diskursforschung. Empirische Analysen zu Bildungs- und Erziehungsverhältnissen (Wiesbaden : VS Verlag), 59-74

Durkheim, Émile. 1894. Les Règles de la méthode sociologique (Paris : Payot)

Foucault, Michel. 2004. Naissance de la biopolitique : Cours au Collège de France (1978-1979) (Paris : EHESS, Gallimard, Seuil)

Foucault, Michel. 2001. Dits et écrits, $\mathrm{n}^{\circ}$ 345, vol. II (Paris : Gallimard)

Goffman, Erving. 1991 [1974]. Les Cadres de l'expérience (Paris : Minuit)

Grinshpun, Yana. 2013a. « Le discours rapporté et le 'discours relais' » : le cas du président et de la princesse », Cahiers de praxématique 57 [En ligne : http://praxematique.revues.org/1765]

Grinshpun, Yana. 2013b. « Discours manifestant et contestation universitaire (2009) », Argumentation et Analyse du Discours10 [En ligne : http://aad.revues.org/1476]

Krieg-Planque, Alice \& Claire Oger. 2010. « Discours institutionnels. Perspectives pour les sciences de la communication », Mots. Les langages du politique 94, «Trente ans d'étude des langages du politique. (1980-2010)», 91-96

Legris, Patricia. 2010. « Les programmes d'histoire en France : la construction progressive d'une « citoyenneté plurielle » (1980-2010)», Histoire de l'éducation 126, 121-154

Lemke, Thomas. 2004. « Marx sans guillemets » : Foucault, la gouvernementalité et la critique du néolibéralisme. Actuel Marx 2, 13-26

Macherey, Pierre. 2012. « Deux figures de l'interpellation : « Hé, vous, là-bas! » (Althusser) «Tiens, un nègre! » (Fanon). Journée d'étude « Le sujet et le pouvoir », [En ligne : https:// philolarge.hypotheses.org/1201]

Maingueneau, Dominique. 1992. «Le tour ethnolinguistique de l'analyse du discours ", Langages 105, 114-125

Maingueneau, Dominique. 2005. « Détachement et surdestinataire. La correspondance entre Pascal et les Roannez », Semen 20 [En ligne : http://semen.revues.org/1086]

Maingueneau, Dominique. 2010. «Le discours politique et son “environnement” ", Mots. Les langages du politique $94,85-90$

Paveau, Marianne.1999. «Le discours officiel des instructions officielles au lycée en 1995 : jeux et enjeux énonciatifs », Pratiques 101-102, 10-20

Marin, Louis. 1993. De la représentation (Paris : Seuil)

Rabatel, Alain. 2015. « Une analyse de discours du manifeste 'Pour des universités à la hauteur de leurs missions'. Pour une alternative à la gestion libérale des universités et de la recherche en Europe », Semen 39 [En ligne : http://semen.revues.org/10477] 
Rayou, Patrick. 2002. La 'dissert de phil'. Sociologie d'une épreuve scolaire (Rennes : P. U. de Rennes)

Ricard-Fersing, Éliane, Marie-José Dubant-Birglin \& Jacques Crinon (2002) : « Mémoires

professionnels et portfolios dans la formation des enseignants. Une étude comparative », Revue française de pédagogie, 139, 121-129

Sarfati, Georges-Elia. 2007. « Note sur "sens commun" : essai de caractérisation linguistique et sociodiscursive ", Langage et société 119, 63-80

Sarfati, Georges-Elia. 2011. « Analyse du discours et sens commun : institution de sens, communauté de sens, doxa, idéologie », Guilhaumou, Jacques \& Philippe Schepens. Matériaux philosophiques pour l'analyse du discours (Besançon : Presses universitaires de Frache-Comté), 139-173

Schön, Donald. A. 1993. Le praticien réflexif, À la recherche du savoir caché dans l'agir professionnel (Montréal : Éditions Logiques)

Wrana, Daniel. 2006. Das Subjekt schreiben. Reflexive Praktiken und Subjektivierung in der Weiterbildung. Eine Diskursanalyse (Baltmannsweiler : Schneider)

\section{NOTES}

1. La sociologie critique renvoie au "conformisme logique» qui alimente les valeurs socioculturelles et les modes de pensée et d'action de la société (Durkheim 1894) mais, contrairement à l'analyse du discours, elle n'analyse pas l'expression langagière des acteurs.

2. Le «sens commun" est défini par Sarfati (2007: 145) comme «ensemble des manières de signifier et savoirs propres aux membres d'une même communauté de sens, en tant qu'ils sont investis dans une même relation d'objet, en vue de fins spécifiques, internes et externes à cette communauté ». Il distingue plusieurs critères pour les caractériser et entre autres, «le statut discursif» qu'il définit comme suit: "l'institution d'une topique (canon) donne lieu à une présentation (exposé) généralement complète de ses normes directrices, à une présentation adaptée (vulgate) à l'occasion d'une transmission (explication). Au-delà, ce sont des normes naturalisées qui retient de leur banalisation (doxa) le domaine public ».

3. «Les inspecteurs généraux ont une mission de conseil et d'orientation des politiques éducatives. Ils se répartissent en quatorze groupes permanents, animés chacun par un doyen : les douze groupes disciplinaires (biologie et géologie, économie et gestion, éducation physique et sportive, enseignements artistiques, histoire et géographie, langues vivantes, lettres, mathématiques, philosophie, physique-chimie, sciences sociales, sciences et techniques industrielles) et deux groupes de spécialité à vocation plus générale (enseignement primaire, établissements et vie scolaire)» (Attali 1995).

4. La question de la relation entre énonciateurs et locuteurs a été explicitement posée à plusieurs reprises par des chercheurs en analyse du discours, p. ex. par Burger, Jacquin et Micheli (2011) ou encore dans un colloque à l'université de Besançon en 2016 (URL : adcost-elliadd.univ-fcomte.fr/ pages/fr/menu4101/les-acteurs-du-discours-18180.html).

5. Voir, pour la question de l'autorité des Programmes, Paveau (1999).

6. http://www.education.gouv.fr/cid60413/publication-de-rapports-des-inspections-generalesigen-igaenr.html.

7. http://www.education.gouv.fr/pid267/les-rapports-igen.html.

8. Les évolutions politiques et culturelles des années 1990 donnent lieu à des programmes modifiés de façon à se centrer davantage sur l'Europe, et enfin, la décennie suivante, au cours de laquelle la citoyenneté à promouvoir devient bien plus complexe. 
9. Qui suppose par exemple la livraison de matériel pédagogique.

10. ESPÉ et jusqu'en 2013, IUFM (Instituts de formation des maîtres).

11. Le cadrage institutionnel est fondé sur différents niveaux institutionnels, le niveau national/ ministériel des Programmes et le niveau académique - en France les sont des instances académiques - ce qui donne lieu à des commandes hétérogènes d'un territoire à l'autre.

12. Ethos personnel : point de vue unique du stagiaire, ethos professionnel : centration sur la préparation de la classe et décisions, ethos scientifique : mise en scène du chercheur / références théoriques en lien avec les leçons professionnelles.

13. Cf. par exemple Burger et al. $(2011: 12)$ : «le discours politique est sous-tendu par la construction d'identités : tout locuteur politique prétend en effet parler au nom d'un collectif, que son discours s'attache à instituer. ».

14. Inspirés par E. Goffman, Brown et Levinson distinguent la face positive (image favorable que l'on tente de donner de soi) et la face négative (liée à la défense d'un « territoire »).

15. https://www.univorleans.fr/sites/default/files/ESPE/documents/ diapo_tuteurs_etab_disci_non_adossees_meef_explicitation_ecrit_reflexif_21_sept_2015.pdf.

D'autre part, les chercheurs font état de la rareté des indices de réflexivité effective dans la plupart des écrits réflexifs décelables au niveau sémantique et énonciatif (Crinon \& Guigne 2006, Ricard-Fersing et al. 2002)

16. Et si la notion de «praticien réflexif » de Schön (1993) est souvent convoquée, elle n'est pas nécessairement mise en œuvre.

17. Captures d'écran. URL: http://forums-enseignants-du-primaire.com/topic/151796-écritréflexif/

18. Ajoutons à cela que l'introduction du mémoire de Master dont il est question n'a en fait pas toujours supprimé «l'écrit réflexif», appellation qui continue de cohabiter avec celle de «portfolio ».

19. Master « Métiers de l'enseignement, de l'éducation et de la formation ».

$20 . \quad$ http://www.cafepedagogique.net/lexpresso/Pages/ 2015/11/25112015Article635840312206230869.aspx

21. Pour le terme d'interpellation voir Macherey (2012) : Si l'idéologie interpelle, c'est en lançant un appel qui s'adresse à tous et auquel tous sans exception, non seulement doivent répondre, mais répondent effectivement, en admettant, en "reconnaissant" [...]. »

22. Cette hypothèse a été confirmée lors d'un sondage auprès d'une vingtaine d'étudiants en formation professionnelle.

23. Cf. Lemke (2004: 20) : "La stratégie qui consiste à rendre les sujets individuels "responsables" [...] mène, en ce qui concerne les risques sociaux comme la maladie, le chômage, la pauvreté, etc., ainsi que la vie en société, à un transfert de responsabilité dans le domaine de la responsabilité $\mathrm{du}$ sujet et transforme ladite responsabilité en un problème de "souci de soi". Cette forme d'individualisation n'apparaît donc pas comme quelque chose qui se trouve hors de l'État. »

24. Les notions de « discours fermé » et de « discours ouvert » aident à observer les modalités de diffusion des discours et le dialogisme à l'œuvre.

25. Les programmes de formation instituent en effet l'enseignement de la littérature à travers l'étude de textes supposés illustrer un trésor patrimonial et transmettre des valeurs fondatrices dans la formation des individus.

26. Cette intervention est précédée de deux interventions sur le même thème en février 2006 alors que N. Sarkozy est ministre de l'intérieur, puis en avril 2008, alors qu'il a été élu Président de la république

27. http://www.dailymotion.com/video/x68n3c_nicolas-sarkozy-s-en-prend-a-la-pri_news

28. La formule désigne la population des travailleurs soumise à des horaires contraignants. 


\section{RÉSUMÉS}

Dans cet article, on cherche à analyser les dynamiques en jeu dans le champ discursif de l'école en France. Pour rendre compte de la diversité des manifestations du dissensus on a examiné des discours contestataires à différents niveaux de la diffusion doxique : rapport interne au ministère de l'éducation nationale, écrits réflexifs de futurs enseignants en formation et intervention politico-médiatique sont des exemples de réaction aux décisions institutionnelles que l'on met en rapport avec la scène d'énonciation dont ils émergent. Les notions de genre discursif, d'ethos et de positionnement sont recrutées pour éclairer les enjeux et les processus de légitimation au fondement des configurations langagières analysées.

This article proposes an analysis of the dynamics at play in the discursive field of school in France. In order to account for the diversity of manifestations of dissent, we examine protest discourses at different levels of doxical diffusion: an internal report in the Ministry of National Education, reflective writings of future teachers during the professional training aswell as a political intervention relayed by the media are examples of reactions to institutional decisions that are related to the scene of enunciation from which they emerge. The notions of discursive genre, ethos and positioning are recruited to enlighten the stakes and processes of legitimization on the basis of the analyzed language configurations.

\section{INDEX}

Keywords : common sense, doxa, dissent, French Discourse Analysis, school discourse

Mots-clés : analyse de discours, discours scolaire, dissensus, doxa, sens commun

\section{AUTEUR \\ PASCALE DELORMAS}

Université Paris-Est, Céditec (EA 3119) 